\title{
Developing Situation and Research Advances of Structural Damage Detection Using BP Network
}

\author{
Jinsheng Fan ${ }^{1,}$ a , Ying Yuan ${ }^{1, b}$ and Xiuling $\mathrm{CaO}^{1, \mathrm{c}}$ \\ ${ }^{1}$ Shijiazhuang University of Economics, Shijiazhuang,Hebei, 050031,China \\ a490371584@qq.com, b275596117@qq.com, ${ }^{\text {c}} 295092204 @ q q . c o m$
}

Keywords: Damage Detection; BP Neural Network; Research Advances

\begin{abstract}
The basic method, research status and development of structural damage identification technology based on BP network are reviewed and summarized, and then the advantages and problems of various methods in theory and practical application are discussed. The development trend and research direction of structural damage identification are proposed, which provides a theoretical basis for structural damage detection.
\end{abstract}

\section{Introduction}

Over the years, the security of the building structure has been a concern of the government departments and the public. At present, many buildings have security risks of different degrees. Once the building collapse, it will bring great loss and disaster. There are many reasons for building damage, but it can be sure that some of the key parts of the building have been seriously beyond the limits of the design. If it can be predicted before the disaster, and the fatigue damage of the building is monitored, and the health status of the building is given, it will greatly reduce the occurrence of accidents. In order to avoid the occurrence of safety accidents and the maintenance cost, the construction health monitoring and damage identification technology are becoming more and more important in the field of civil engineering.

At present, there are many methods for damage detection of structures, but most of them are based on experimental research methods by measuring the displacement or acceleration of structure and other indicators, even in some cases it will cause a certain degree of damage to the structure. Artificial neural network is a new developing subject in 1990s, which is a kind of nonlinear dynamic network structure built by simulating human's brain, and it is a complex network system, in which a number of simple neurons are connected in a certain way. According to the characteristics of the input sample space, the system can automatically find the nonlinear rules between input and output, and establish a mathematical model which can accurately reflect the inherent law of the input and output data, so as to estimate and evaluate the target accurately. At present, artificial neural network is widely used in the field of structural damage detection.

\section{Basic working principle of BP neural network for damage identification}

There are many kinds of artificial neural network (ANN) system. The BP (Back Propagation) neural network is one of the most widely used neural networks. It is not only the core of the feed forward network, but also the basis of other types of neural networks.

BP neural network is widely used in structural analysis because of its strong nonlinear mapping ability, and it is usually constructed with three layers, namely, one input layer, one hidden layer and one output layer. The basic idea is: first, the measurement data is used to train the network, the network parameters are determined by using the appropriate method. Then the input data is sent to the network. The network has the corresponding output. If the learning process is successful, the output of the system and the output of the network should be consistent with the characteristics of the system. On the contrary, when the system is damaged, there is a difference between the output of the system and the output of the network, this difference is a measure of damage. So the nonlinear mapping relationship between the structural model parameters and the results of the modal parameters can be 
established by the neural network, and then by the measured model parameters of the structure and the nonlinear mapping relationship, the physical, geometrical and boundary conditions of the structure are obtained, and the structural dynamic model modification is completed.

The structural damage identification based on neural network mainly includes the following steps: determination of network structure, selection of parameters, study sample size, selection of initial weights and structure damage detection. According to this idea, the modal parameters obtained from the measurement are inputted into the trained neural network model, and the variation of structural parameters can be obtained, and the location and degree of the structural damage can be identified.

A classic 3 layer BP network [1] topology is shown in Fig.1. $\mathrm{X}_{\mathrm{i}}$ is input layer, $\mathrm{Z}_{\mathrm{k}}$ is hidden layer, $Y_{j}$ is output layer. The purpose of the network study is to use the error between the actual output and the target of the network modifying the network weights and threshold values by gradient descent method, to achieve the minimum of the Error sum of squares of the network output layer, so that the output in theory gradually close to the target.

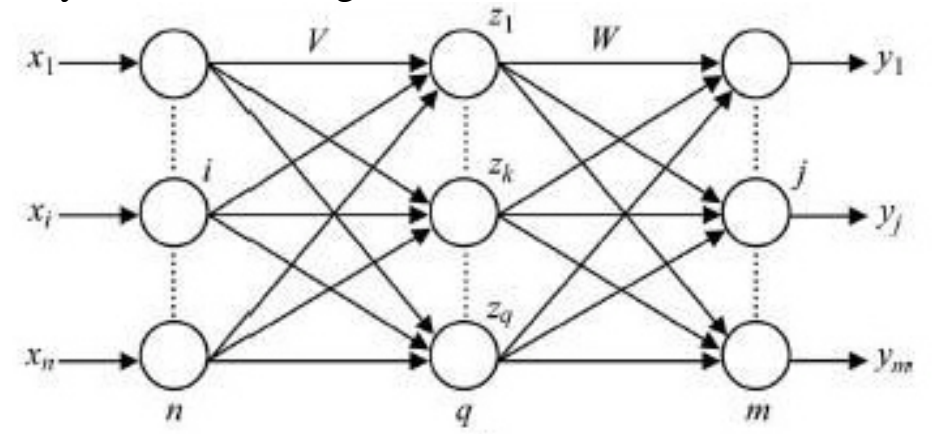

Fig.1 Topology diagram for multi-layer feedforward neural network

\section{Research status of structural damage identification based on BP network}

BP neural network is widely used in neural network, which is widely used in the field of civil engineering. Foreign scholars have made a lot of achievements in the research of structural damage identification based on BP neural network. Venkatasubramanian and Chan [2] used BP neural network to carry out the diagnosis and identification of structural damage in 1989. Tsou [3] took the change of natural frequency and the change of dynamic residual vector as the input of BP neural network to detect damage, and the validity and applicability of the proposed method were demonstrated by using a spring proton system. Kudva [4] studied the use of BP network method for damage identification of flat plate structures;

Many domestic scholars have started to pay attention to the neural network for structural damage identification in the early twentieth century, and a lot of researches have been carried out. SUwen Chen[5] proposed a multi-hierarchical damage identification approach based on BP network for frame structures, the main idea of this approach is described as follows. Firstly, the damaged story is detected. Then what ought to do is to identify the damaged member's location among the members in the detected story, finally, the damaged members' severity is identified. Employing this approach, the number of training samples can be reduced greatly. Another advantage is that the approach has high precision. Application to a 3 layers steel frame structure subjected to earthquakes simulated by shaking table has demonstrated the effectiveness of this approach.

Jie Yang[6] calculated 520 cable-damage cases of the Runyang cable-stayed Bridge (North Bridge) crossing the Yangtze River. By defining a normalized natural frequency, one may find the location and extent of cable damage with respect to the changes on the normalized nature frequency. A BP neural network describing the 7-dimension mapping space of damage pattern was founded. The neural training and identification based on the different damage cases validated the method, and an evaluation number of identification error was defined to quantify the identification effect.

Chengji Li[7] put forward a structural damage identification method based on BP network, and introduces particle swarm optimization model to optimize BP network weight measurement and threshold value, set up complete model and damage model, and made a simulation. Through sample 
testing, it proves the BP network optimized by particle swarm optimization model can recognize the location and degree of structural damage, which is rapid and efficient structural damage identification method.

XUhua Deng[8] investigated damage identification of reinforced concrete frame structures by using the changing ratio of modal strain energy and BP neural network. The changing ratio of modal strain energy MSECR is taken as the damage location factor. Then, BP neural network is used to determine the damage degree. The results show that the proposed method can be applied to the damage detection of frame structures. The numerical model is shown as fig.2.

Dong Li[9] used BP neural network to study the damage position and degree for simply supported beam. Firstly, the finite element simulation software was used to calculate the first two natural frequencies of the structures with different damage and the curvature mode of the specially appointed points; Then they were made as the input of a BP network for simply supported beam's damage identification; finally, the trained network detects the damage. The results show that; this method can effectively identify the damage position there will be a high accuracy for the identification of damage degree.

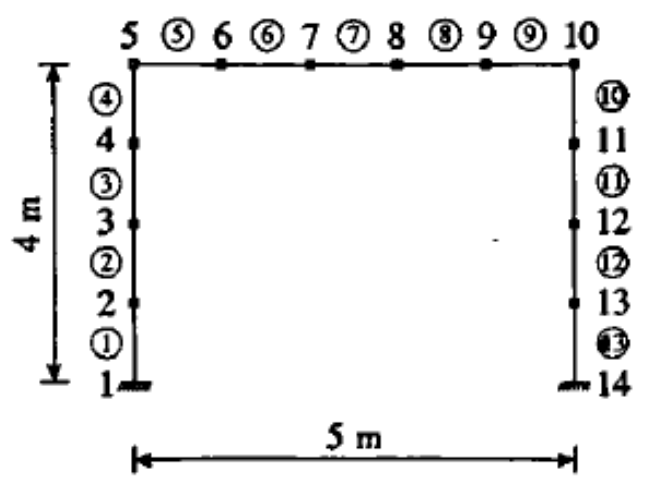

Fig.2 Finite element model for frame

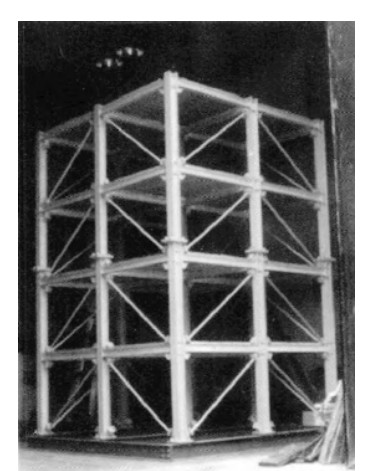

Fig.3 Benchmark experiment model

Hong Chang[10] studied the damage degree of the Benchmark model established by the structural health monitoring task force of the American Civil Engineers Association. The model is a four story steel frame structure, and the longitudinal and transverse is both two spans. It takes natural frequency change ratio as the characteristic parameters of the network and the load of the model is simulated by wind load, then BP neural network model is used to be trained and simulated. Results prove it is feasible, and the accuracy is high. Its laboratory 3D model is shown as fig.3.

In view of the problems of incomplete modal information, modal density and large degree of freedom in the damage identification of reticulated shell structures, Jing $\mathrm{Xu}$ [11] proposed a damage identification method based on time series auto regressive model (AR model) and BP neural network. Take the Kewitte single-layer spherical reticulated shell as an example, using the method to identify damage in the rod position. Numerical simulation results show that the method has high accuracy and the anti noise performance.

\section{Key issues for current research}

Structure damage identification technology based on BP neural network is a research subject with strong engineering application background. This technology has good application prospect in structural damage identification and diagnosis. But there are some problems need to be solved :(1) at present, the structural type for research is diverse, and the structure model is developed from 2D to $3 \mathrm{D}$, which is prone to modeling errors. The main causes of the model error are the discrete of material properties, the simplification of the boundary conditions, the incorrect modeling of the non structural components.

(2) If there are more than two damage elements, the workload of network training will be very large, for more complex structures, it is likely to have a sample explosion. If the damage location can 
be identified firstly, it can greatly reduce the number of training samples and solve the problem of sample combination explosion.

\section{Conclusion}

The following aspects may have important significance in the future development: (1) Application of damage identification technology based on hybrid model has important practical value for damage identification of civil engineering structures. It can overcome the difficulty that the number of training samples is huge only using BP neural network by combining other neural network with it.

(2) It is more practical to extract the feature parameters of the damage structure by the natural incentive form. For some kind of structural damage form, it is necessary to find the proper natural incentives, which can get the response of the structure fully demonstrating the damage.

(3) In order to obtain a good result, we must choose the reasonable testing time to reduce the environmental noise, and give priority to the structural damage identification method based on statistical model, in order to reduce the error caused by data analysis.

\section{Acknowledgements}

This work was financially supported by Natural Guidance Project of Hebei Province Department of Education (Z2012137).

\section{References}

[1] Zengren Yuan: Artificial neural network and its application (Tsinghua university press, China 1999)

[2] Venkatasubramanian V and Chan K: Journal of AIChE.Vol.35 (1989), p.1993

[3] Tsou P and Shen M-HH: AIAA Journal. Vol.32 (1994), p.176

[4] Kudva, C. Surace: Journal of Sound and Vibration. Vol.206 (1997), p.567

[5] S.W.Chen and G.Q.Li: J.Earthquake engineering and engineering vibration In Chinese. Vol.22 (2002), P.18

[6] J.Yang, A.Q.Li and C.Q.Miu: J. China civil engineering journal In Chinese. Vol.39 (2006), p.72

[7] C.j.li, G.Y.He and X.J.Zhang: J.Shanxi architecture In Chinese. Vol.35 (2009), p.91

[8] X.H.Deng and X.S.Tang: Journal of transport science and engineering In Chinese.Vol.26 (2010), p.53

[9] D.Li, C.P.Zeng and K.Ma: Journal of Guizhou University In Chinese. Vol. 30(2013), p.110

[10] H.Chang and B.Zhang: Journal of Jilin Institute of Architecture \& Civil Engineering In Chinese. Vol.31 (2014), p.23

[11] J.Xu, W.Guo and X.L.Wang:J. Spatial structures In Chinese. Vol.21 (2015), p.66 